\title{
ALGORITMOS PMV-MBPC PARA CONFORTO TÉRMICO EM EDIFICAÇÕES E APLICAÇÃO EM UMA CÉLULA-TESTE
}

\author{
Emerson Donaisky* \\ emerson.donaisky@pucpr.br \\ Nathan Mendes ${ }^{\ddagger}$ \\ nathan.mendes@pucpr.br \\ ${ }^{*}$ CCET \\ ${ }^{\dagger}$ PPGEPS/CCET \\ ${ }^{\ddagger} \mathrm{PPGEM} / \mathrm{CCET}$ \\ Pontifícia Universidade Católica do Paraná (PUCPR) \\ Rua Imaculada Conceição 1155 CEP 80215-901 \\ Curitiba - PR, Brasil
}

Gustavo H. C. Oliveira ${ }^{\dagger}$

gustavo.oliveira@pucpr.br

\begin{abstract}
PMV-MBPC Algorithms for Thermal Comfort: An Application to a Test-cell

The present paper is focused on thermal comfort control problem for building occupants. Thermal comfort is a concept difficult to define and, here, the PMV (Predicted Mean Vote) index is used by means of two predictive control strategies, characterized by having terminal constraints, called here PMV-MBPC (PMV Model Based Predictive Controller). The first thermal comfort control strategy is based on generating a temperature set-point signal that optimizes the building (single zone) internal PMV value. The second one includes the PMV model in the controller prediction computations, generating a non-linear PMV model having Wiener structure. Results related to closed loop system stability are proposed. In this context, an environment for control systems tests is described and the first approach is then implemented in real time using an oil-heater and this environment. Experimental results illustrate the thermal comfort control system performance. Additionally, simulation
\end{abstract}

Artigo submetido em 25/03/2009 (Id.: 00977)

Revisado em 20/04/2009, 15/07/2009

Aceito sob recomendação do Editor Associado Prof. José Roberto Castilho Piqueira results, conducted with hourly weather data, also illustrate the control algorithms performance.

KEYWORDS: Thermal Comfort, PMV, Predictive Control, Optimization.

\section{RESUMO}

Este artigo trata o problema de controle de conforto térmico para ocupantes de edificações. Conforto térmico é um conceito de difícil definição e, neste trabalho, utiliza-se o índice PMV (Predicted Mean Vote) para sua avaliação. Através deste índice, duas estratégias de controle preditivo caracterizadas por ter restrições terminais, denominadas aqui de PMV-MBPC (PMV Model Based Predictive Controller), são apresentadas. Na primeira estratégia, a gestão do conforto térmico é realizada através da geração de sinais de referência para o controlador, que otimiza o valor de PMV dentro de uma zona térmica da edificação. Na segunda, o modelo de PMV está incluso nos cálculos de previsão do controlador, gerando um modelo não-linear com estrutura Wiener. Resultados relacionados com a garantia de estabilidade do sistema em malha fechada são propostos. Neste contexto, um ambiente para testes (célula-teste) de sistemas de controle é descrita e a primeira abordagem é então implementada em 
tempo real neste ambiente usando um aquecedor a óleo. Resultados experimentais ilustram o desempenho do sistema controle para conforto térmico. Adicionalmente, resultados de simulação, conduzidos com dados climáticos horários, ilustram também o desempenho dos algoritmos de controle.

PALAVRAS-CHAVE: Conforto Térmico, PMV, Controle Preditivo, Otimização.

\section{INTRODUÇÃO}

Eficiência energética nos setores residencial, comercial, industrial e de transportes tem uma grande importância atualmente devido às grandes crises ambiental e econômica.

Muitos fatores influenciam no aumento do consumo de energia. Entre estes, os principais são: os padrões de vida da população e as condições meteorológicas. É comum em climas quentes ou frios, o consumo de energia aumentar para fins ou de aquecimento ou de arrefecimento (Erbe, 2006). Sistemas de climatização estão presentes nos setores residenciais e comerciais que, por sua vez, representam uma parcela significativa do consumo total de energia (Erbe, 2006; FURNAS, 2006). A utilização de sistemas de condicionamento de ar vem crescendo progressivamente nos países em desenvolvimento e, segundo Salsbury (2006), são responsáveis por até um terço da energia total consumida em países desenvolvidos. Na França, sistemas de climatização são responsáveis por $40 \%$ do consumo de energia fóssil do país (Raffenel et al., 2008). Portanto, a relevância deste problema tem atraído progressivamente a atenção das pesquisas acadêmicas e industriais desde a década de 1970 e a automação é uma ferramenta para melhorar a eficiência energética em edificações (Castro et al., 2005).

De fato, cada vez mais a população vem passando um tempo significativo de suas vidas em ambientes climatizados artificialmente e a lacuna entre esta climatização e o conforto térmico tem efeitos diretos na produção e na satisfação de cada indivíduo. Se os ambientes de trabalho não oferecem conforto térmico, o desempenho dos trabalhadores diminui. Conseqüentemente, o foco é manter o conforto térmico dos ocupantes reduzindo o gasto de energia. O setor residencial, que responde por uma parcela significativa do consumo de energia e, economias nesse consumo, podem ser obtidas através de melhorias no sistema de controle de climatização (Erbe, 2006). Já no setor comercial, a palavra chave é a automação da edificação, que passa pelo controle eficiente dos sistemas de climatização. Segundo Georgiev (2006), aproximadamente $30 \%$ do consumo de energia pode ser reduzido através de um controle mais eficiente dos sistemas de climatização. A utilização de sistemas de controle em automação predial é abordada também por Luna Filho and Bauchspiess (2003).
Por outro lado, conforto térmico em edificações é um conceito que é difícil de se definir. Nas últimas décadas, um grande número de índices foi estabelecido para análise de climas em ambientes fechados e projetos de algoritmos de controle para sistemas de climatização ou HVAC (Heating, Ventilating and Air Conditioning) (Fanger, 1970; Sherman, 1985; Gagge et al., 1986; ASHRAE, 2001). No contexto do Brasil, este problema é abordado por Xavier and Lamberts (2000). Em comum tem-se o fato que medir conforto térmico não está restrito à medição de temperatura. Fanger (1970), por exemplo, propôs um método para estimar níveis de conforto térmico que, além da temperatura e da umidade relativa, inclui temperatura radiante média, velocidade do ar e fatores individuais como taxa metabólica e resistência térmica da vestimenta. Um índice baseado nessas variáveis, o PMV (Voto Médio Estimado ou Predicted Mean Vote), é obtido e quanto mais próximo de zero for o valor do PMV, melhor será a sensação de conforto térmico dos ocupantes.

Muito tem-se estudado sobre controle de sistemas de climatização, porém este problema é tratado apenas como um controle de temperatura, desprezando-se a influência de outros parâmetros no conforto térmico. Entretanto, com esta estratégia, mesmo quando aplicada em novos prédios comerciais equipados com modernos sistemas de climatização, podese encontrar indivíduos insatisfeitos em relação ao conforto térmico (Muzi et al., 1998).

Algoritmos de controle para conforto térmico em sistemas de climatização são aqueles que consideram, explicitamente na estrutura da lei de controle, aspectos relacionados com conforto térmico, além da simples realimentação de temperatura. Um exemplo é admitir o PMV como um sinal mensurável e controlável, e incluí-lo como parte da realimentação em uma estrutura de malha-fechada. Alguns exemplos são os trabalhos de Kolokotsa et al. (2001) e Gouda et al. (2001), no contexto de algoritmos de controle PID (Proporcional, Integral e Derivativo) e Fuzzy. Por outro lado, o índice PMV pode ser incluído na função custo para gerar uma lei de controle de conforto térmico baseado nos fundamentos do MBPC (Model Based Predictive Control ou Controle Preditivo Baseado em Modelo) (Freire et al., 2005a; Freire et al., 2005b; Freire et al., 2008; Donaisky et al., 2007; Donaisky et al., 2008; Donaisky, 2008).

No presente artigo, duas estratégias de controle de conforto baseadas no índice PMV e no MBPC (denominadas aqui de PMV-MBPC) são propostas e comparadas. Na primeira, tendo como base uma malha de controle com algoritmo CRHPC (Constrained Receding Horizon Predictive Control)(Camacho and Bordons, 1999), o índice PMV é utilizado em uma malha de supervisão para geração de um sinal ótimo de referência. Resultados em tempo real em uma 
célula-teste de $8 m^{3}$ com condições higrotérmicas externas monitoradas, são apresentados. Na segunda, o algoritmo PMV é incorporado no modelo de previsão formando uma estrutura não-linear do tipo Wiener. O algoritmo de controle resultante é não-linear com com restrições terminais ou NCRHPC (Non-linear CRHPC). Resultados relacionados com a estabilidade do sistema em malha fechada com este controlador não-linear são propostos.

O artigo está organizado como se segue. Na próxima seção, questões de modelagem da edificação são apresentadas. A Seção 3 contém os dois algoritmos PMV-MBPC propostos no presente artigo. $\mathrm{Na}$ Seção 4 , resultados de simulação são mostrados e discutidos e, na Seção 5, a estratégia de controle de conforto térmico é aplicada em tempo real. Finalizando, na Seção 6, as conclusões são apresentadas.

\section{DETALHES DA EDIFICAÇÃO QUESTÕES DE MODELAGEM}

Uma edificação pode ser vista como um sistema dinâmico multi-variável onde os principais sinais de entrada são o sinal de controle enviado ao equipamento de climatização, a temperatura externa, a umidade relativa externa e a radiação solar global. Os sinais de saída são a temperatura e umidade relativa internas. Embora uma edificação seja um sistema não-linear, principalmente devido às relações termodinâmicas presentes para o ar úmido, usualmente modelos lineares são capazes de fornecer uma representação adequada da dinâmica do sistema. Um sinal de saída relevante, relacionado com o problema tratado no presente artigo, é o sinal de PMV. Esta saída é obtida usando informações climáticas relativas ao ambiente e parâmetros individuais dos ocupantes, uma vez que o PMV pode ser descrito pelo mapeamento não linear como apresentado na Eq. 1:

$$
P M V=G\left(T, \phi, T_{\mathrm{rm}}, V, M, I_{\mathrm{cl}}\right),
$$

onde os parâmetros ambientais são a temperatura $T$, a umidade relativa $\phi$, a temperatura radiante média $T_{\text {rm }}$ e a velocidade do ar $V$. Os parâmetros individuais são os índices de atividade $M$ e vestimenta $I_{\mathrm{cl}}$. Mais detalhes sobre o mapeamento do PMV podem ser encontrados em Fanger (1970), Kon (1994), Trebien et al. (2007) e Donaisky (2008). Cada atividade física está relacionada a uma taxa metabólica. Taxas metabólicas e o cálculo dos respectivos índices (met) podem ser estimados por meio de análise do balanço termodinâmico de um indivíduo (Prek, 2005; Prek, 2006) ou por meio de medições realizadas direta ou indiretamente. A obtenção direta consiste em medir calorimetricamente a energia térmica liberada por uma pessoa, enquanto a indireta é obtida por meio da medição de volume de ar aspirado e de níveis de oxigênio e gás carbônico expirado (Metabolic Rate Calculator, 2009). Por outro lado, Já o índice de vestimenta está baseado nas propriedades medidas ou estimadas da condutividade (ou resistência) térmica de cada tipo de roupa, levando-se em conta uma superfície média de troca de calor de $1,7 \mathrm{~m}^{2}$. Normas e estudos sobre estas análises podem ser encontrados em ISO 9237 (1995), Meinander (1985) e Meinander (1994). Uma tabela relacionando índices para cada tipo de atividade e cada tipo de roupa pode ser encontrada em Clothing and Thermal Insulation (2009) e Clothing, Activity and Human Metabolism (2009). Um exemplo de software que compila estas tabelas é o Analysis CST (2009).

Um resumo das informações mais usuais que relacionam as atividades e vestimentas com os respectivos índices é apresentada em Tab. 1 e Tab. 2.

Tabela 1: Relação entre Tipo de Atividade e Metabolismo.

\begin{tabular}{|c|c|}
\hline Tipo de Atividade & Metabolismo (met) \\
\hline Deitado & 0,8 \\
\hline Sentado descansando & 1,0 \\
\hline Sentado com atividade leve & 1,2 \\
\hline Em pé com atividade leve & 1,6 \\
\hline Em pé com atividade média & 2,0 \\
\hline Atividade intensa & 3,0 \\
\hline
\end{tabular}

Tabela 2: Relação entre Vestimenta e Resistência Térmica.

\begin{tabular}{|c|c|}
\hline Vestuário & $\begin{array}{c}\text { Resistência } \\
\text { Térmica }(\mathrm{clo})\end{array}$ \\
\hline Somente calção & 0,1 \\
\hline Vestuário tropical & 0,3 \\
\hline Vestuário leve de verão & 0,5 \\
\hline Vestuário para trabalho & 0,7 \\
\hline Agasalho leve & 1,0 \\
\hline Traje completo & 1,5 \\
\hline
\end{tabular}

O índice PMV pode variar de -3 (frio) $\mathrm{a}+3$ (quente) e quanto mais próximo de zero estiver o valor de PMV, melhor será a sensação de conforto térmico dos ocupantes. A Tab. 3 contém a relação entre o valor do PMV e a sensação térmica do indivíduo. Também nesta tabela está apresentado o índice PPD, que é a porcentagem estimada de indivíduos insatisfeitos no ambiente.

O diagrama de blocos de todo o sistema é apresentado na Fig. 1. Do ponto de vista da modelagem, dois sistemas são 
Tabela 3: Relação entre PMV, PPD e sensação térmica.

\begin{tabular}{|c|l|c|}
\hline PMV & Sensação Térmica & PPD (\%) \\
\hline+3 & Muito Quente & 100 \\
\hline+2 & Quente & 75 \\
\hline+1 & Levemente Quente & 25 \\
\hline 0 & Neutra & 5 \\
\hline-1 & Levemente Frio & 25 \\
\hline-2 & Frio & 75 \\
\hline-3 & Muito Frio & 100 \\
\hline
\end{tabular}

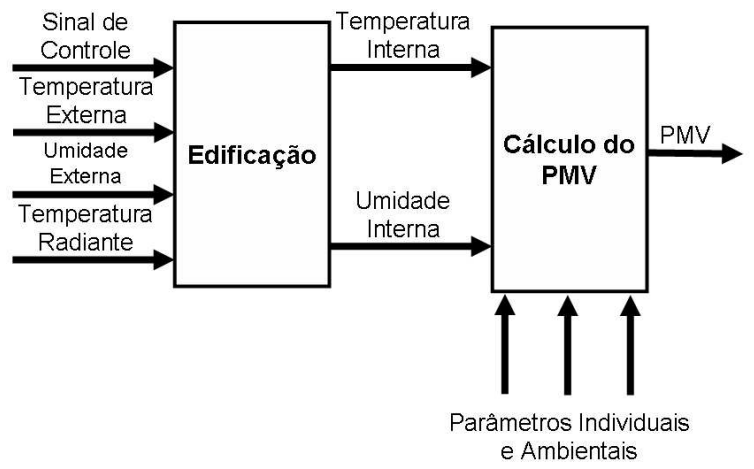

Figura 1: Diagrama de blocos de uma edificação.

tratados no presente artigo para a síntese da lei de controle em malha-fechada. Estes modelos são usados para o desenvolvimento das equações de previsão para os algoritmos de controle da próxima seção.

O primeiro é um modelo linear relacionando o sinal de controle e o sinal de temperatura interna. Portanto, uma equação de previsão de temperatura $j$-passos à frente pode ser construída, e será representada aqui por $\hat{y}_{\mathrm{T}}(k+j \mid k)$.

O segundo é um modelo não-linear, estrutura Wiener, relacionando o sinal de controle com o sinal de PMV, como apresentado na Fig. 2. O procedimento é similar aos mencionados previamente e as previsões serão representadas aqui por $\hat{y}_{\mathrm{T}}(k+j \mid k)$ e $\hat{y}_{\phi}(k+j \mid k)$. Quando a velocidade do ar e os parâmetros individuais são assumidos constantes e $T_{\mathrm{rm}}=T$, tem-se a equação de previsão:

$$
\begin{aligned}
\hat{y}_{\mathrm{PMV}}(k+j \mid k)= & G\left(\hat{y}_{\mathrm{T}}(k+j \mid k), \hat{y}_{\phi}(k+j \mid k),\right. \\
& \left.\hat{y}_{\mathrm{T}}(k+j \mid k), V, M, I_{\mathrm{cl}}\right) .
\end{aligned}
$$

Assumir que $T_{\mathrm{rm}}=T$ é equivalente dizer que a temperatura média das superfícies das paredes internas são iguais a temperatura interna do ar. Em Trebien et al. (2007) o problema

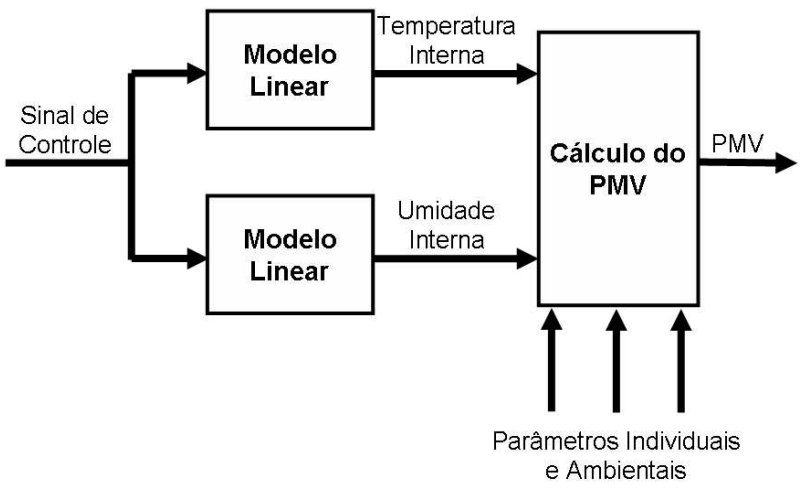

Figura 2: Modelo de PMV não-linear.

de medição de PMV em sistemas de controle é analisado. Neste trabalho, conclui-se que um erro de $4^{\circ} \mathrm{C}$ nesta hipótese implica um erro de, no máximo, 0,5 no valor final do PMV calculado.

\section{LEIS DE CONTROLE PREDITIVO PARA CONFORTO TÉRMICO}

Controladores preditivos baseados em modelo são definidos pelos seguintes passos: $i$ ) um modelo é usado para computar as previsões de saída do processo; $i$ ) uma função custo relacionada com o desempenho do sistema em malha-fechada é definida; iii) esta função custo é minimizada em relação ao conjunto de futuros sinais de controle; iv) o primeiro destes sinais de controle ótimos é aplicado no processo, i.e., a estratégia horizonte rolante. Neste contexto, uma classe de algoritmos de controle preditivo inclui, na otimização da função custo, restrições nos valores finais da previsão de saída. A principal razão para essas restrições é garantir estabilidade em malha-fechada sem a necessidade de usar horizontes infinitos na função custo.

O presente artigo expande os trabalhos de Freire et al. (2005b), Freire et al. (2008), Donaisky et al. (2007), Donaisky et al. (2008) e Donaisky (2008) propondo dois algoritmos MBPC para o controle de conforto térmico baseado em PMV (PMV-MBPC). Os algoritmos são caracterizados pelos seguintes pontos: $i$ ) a entrada do processo é representada por um simples equipamento de climatização (um sinal de controle) e dois sensores internos para medir a temperatura e a umidade relativa; ii) o índice PMV é considerado para promover conforto térmico aos ocupantes. A principal diferença entre eles é: um método usa um cálculo baseado no PMV para gerar a referência de temperatura para a lei de controle e o outro usa as equações de PMV para obter um modelo não-linear. Tal modelo é usado então com a finalidade de previsão. Estes métodos são apresentados na seqüência. 


\subsection{MBPC com modelo interno de PMV}

Este método é caracterizado por usar um modelo em espaço de estados não linear, com estrutura Wiener, para representar o comportamento do PMV. A função custo deste controlador MBPC é:

$$
\begin{aligned}
J_{1}(k)= & \sum_{j=1}^{N_{\mathrm{y}}} \mu(j) \hat{y}_{\mathrm{PMV}}(k+j \mid k)^{2}+ \\
& +\sum_{j=0}^{N_{\mathrm{u}}-1} \lambda(j) \Delta u(k+j \mid k)^{2}
\end{aligned}
$$

onde $N_{\mathrm{y}}$ e $N_{\mathrm{u}}$ definem os horizontes de previsão e de controle, respectivamente; $\lambda(j)$ é um fator de ponderação no sinal de controle; $\mu(j)$ é um fator de ponderação no sinal de saída; $\Delta u(k+j \mid k)$ é o sinal de variação de controle no tempo $k+j$ calculado no tempo $k$. O sinal de referência é igual a zero visto que, quanto mais próximo de zero estiver o índice PMV, melhor será a sensação térmica. $\hat{y}_{\mathrm{PMV}}(k+j \mid k)$ é a previsão no instante de tempo $k+j$, usando o modelo Wiener, conforme Eq. 2.

A lei de controle é obtida minimizando a função custo (Eq. 3) em relação a futuras variações no sinal de controle $\Delta u(\cdot)$, sujeito a restrições terminais e nos sinais de entrada e saída do processo, isto é:

$$
\begin{gathered}
\min _{\Delta u(k \mid k), \Delta u(k+1 \mid k), \ldots, \Delta u\left(k+N_{\mathrm{u}}-1 \mid k\right)} J(k) \\
\text { sujeito a } \\
\Delta u(k+j \mid k)=0 \quad \forall j=N_{\mathrm{u}}, \ldots, N_{\mathrm{y}} \\
\hat{y}\left(k+N_{\mathrm{y}}+j \mid k\right)=w \quad \forall j=1, \ldots, m \\
0 \leq u(k+j \mid k) \leq u_{\max } \quad \forall j=0, \ldots, N_{\mathrm{u}}-1 \\
y_{\min } \leq \hat{y}(k+j \mid k) \leq y_{\max } \quad \forall j=1, \ldots, N_{\mathrm{y}} \\
\operatorname{com} J(k)=J_{1}(k), \hat{y}(k+j \mid k)=\hat{y}_{\mathrm{PMV}}(k+j \mid k) \text { e } w=0 .
\end{gathered}
$$

Esta função custo define um problema de programação nãolinear, com restrições não-lineares. O primeiro conjunto de restrições é relacionado a definição do horizonte de controle e o segundo conjunto implementa as restrições terminais sobre uma janela de restrição $m$. O terceiro e quarto conjuntos asseguram a optimalidade da lei de controle na presença de restrições no sinal de entrada, comum nos equipamentos de climatização ( $u_{\max }$ é o valor máximo do sinal de controle), e no sinal de PMV. Limites comuns para o PMV podem ser definidos como sendo - 0,5 e 0,5 , uma vez que estes valores são considerados limites para a sensação de conforto medido através do PMV.

O conjunto ótimo de futuros sinais de controle pode ser

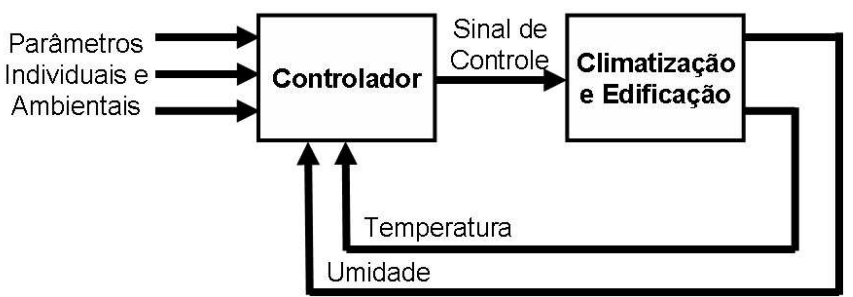

Figura 3: MBPC não-linear com modelo interno de PMV.

obtido usando um algoritmo de Programação Quadrática Seqüencial e o sinal de controle ótimo, i.e., $\Delta u(k \mid k)$, é calculado usando a primeira variação ótima de controle do seguinte modo: $u(k)=u(k-1)+\Delta u(k \mid k)$. A Fig. 3 ilustra a estratégia.

\subsubsection{Estabilidade no MBPC com modelo interno de PMV}

Resultados sobre a estabilidade do sistema em malha fechada para a lei de controle MBPC com restrições terminais para o caso de sistemas lineares foram apresentados na literatura (Scokaert and Clarke, 1994). A seguir, estes resultados, válidos para horizonte finito e baseados na prova da monotonicidade da função custo, são aqui estendidos para o caso não-linear de controle de PMV.

Teorema 3.1 Seja o sistema em malha-fechada, cujo processo é dado pela equação 2 , sem perturbação, e a lei de controle dada pelas equações 3 e 4, sem as restrições de amplitude nos sinais de entrada e saída, o MBPC com modelo interno de PMV se estabiliza se

$$
\begin{array}{ll}
\mu\left(N_{\mathrm{y}}\right) \geq \mu\left(N_{\mathrm{y}}-1\right) \geq \ldots \geq \mu(1), & \mu \neq 0, \\
\lambda\left(N_{\mathrm{u}}-1\right) \geq \lambda\left(N_{\mathrm{u}}-2\right) \geq \ldots \geq \lambda(0) & \text { e } \quad m=n
\end{array}
$$

sendo $n$ a ordem do sistema, acrescida de uma unidade.

\section{Prova:}

A prova está baseada em mostrar que $J(k+1)<J(k)$. Portanto, o critério de custo é monotonicamente decrescente ao longo do tempo, o que garante a estabilidade do sistema em malha fechada.

Seja $J^{*}(k)$ o valor ótimo do critério no instante de tempo $k$, sendo o sinal de controle ótimo dado por $\Delta u^{*}(k+j \mid k)$, $j=0, \ldots, N_{\mathrm{u}}$. Assume-se um valor para o critério de custo próximo instante de tempo $k+1$, isto é, $J_{\mathrm{c}}(k+1)$, calculado com um sinal de controle sub-ótimo dado por $\Delta u_{\mathrm{c}}(k+1+$ $j \mid k+1), j=0, \ldots, N_{\mathrm{u}}$ cujos elementos são iguais aos de 
$\Delta u^{*}(\cdot)$, isto é, $\Delta u_{\mathrm{c}}(k+1+j \mid k+1)=\Delta u^{*}(k+j \mid k) \mathrm{e}$ $\Delta u_{\mathrm{c}}\left(k+N_{\mathrm{u}} \mid k+1\right)=0$.

Portanto, a variação do valor do critério de custo entre $J^{*}(k)$ e $J_{\mathrm{c}}(k+1)$, sabendo que a escolha do vetor de sinais de controle faz com que o conjunto de previsões do PMV para cálculo de $J^{*}(k)$ e $J_{\mathrm{c}}(k+1)$ sejam iguais, que $\hat{y}_{\mathrm{PMV}}\left(k+N_{\mathrm{y}}+1 \mid k\right)=0$ devido à restrição no valor terminal da previsão, tem-se:

$$
\begin{aligned}
& J^{*}(k)-J_{\mathrm{c}}(k+1)=\mu(1) \hat{y}_{\mathrm{PMV}}^{2}(k+1 \mid k) \\
& +\sum_{j=2}^{N_{\mathrm{y}}}[\mu(j)-\mu(j-1)] \hat{y}_{\mathrm{PMV}}^{2}(k+j \mid k) \\
& +\lambda(0)\left(\Delta u^{*}(k \mid k)\right)^{2} \\
& +\sum_{j=1}^{N_{\mathrm{u}}-1}[\lambda(j)-\lambda(j-1)]\left(\Delta u^{*}(k+j \mid k)\right)^{2}
\end{aligned}
$$

o que implica em que $J_{\mathrm{c}}(k+1)<J^{*}(k)$. Como $J_{\mathrm{c}}(k+1) \leq$ $J^{*}(k+1)$, então $J^{*}(k+1)<J^{*}(k)$ e o critério de custo é monotonicamente decrescente ao longo do tempo.

Adicionalmente, se $m=n$, tem-se que a restrição de igualdade para a previsão da saída $\hat{y}_{\mathrm{PMV}}(k+j \mid k)$ em $N_{\mathrm{y}}+1 \leq$ $j \leq N_{\mathrm{y}}+m$ garante que o valor do PMV previsto será também zero também para $j>N_{\mathrm{y}}+m$. Sendo $\mu(1) \neq 0$, o critério de custo do sistema em malha fechada tende a decrescer enquanto o PMV previsto não for nulo.

\subsection{MBPC com modelo externo de PMV}

Na seqüência, uma estratégia de controle de conforto caracterizada pela presença de uma malha externa que calcula, em tempo real, o valor de temperatura que fará o índice PMV ir à zero é apresentado. Este bloco usa informações individuais e ambientais, tais como a umidade relativa medida $y_{\phi}(k)$, a velocidade do ar $V$, a taxa metabólica $M$ e o índice de vestimenta $I_{\mathrm{cl}}$ para gerar o valor desejado de temperatura, do seguinte modo (ver também a Eq. 2):

$$
T^{*}(k)=\arg \min _{T(k)} G\left(T(k), y_{\phi}(k), T(k), V, M, I_{\mathrm{cl}}\right)^{2}
$$

sujeito a

$$
-3<G(\cdot)<3
$$

Este é um problema de otimização não-linear com uma variável, usando-se o valor de temperatura interna medida para iniciar o procedimento.

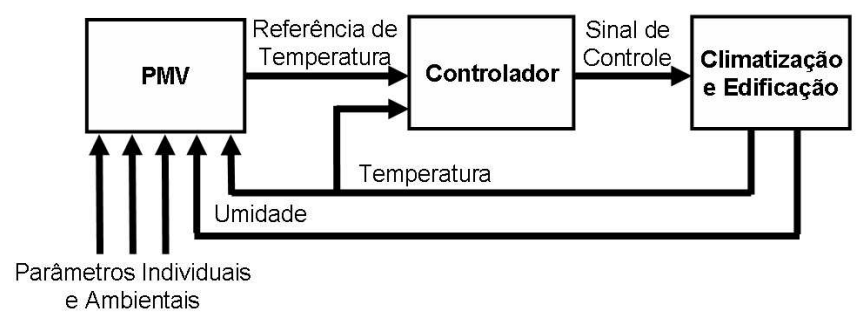

Figura 4: MBPC linear com modelo externo de PMV.

O gerador de referência ótima é utilizado junto com um algoritmo MBPC linear para controle de temperatura. Isto é, o valor ótimo de temperatura interna calculado pelo uso da Eq. 6 é usado como referência para o controle de temperatura no instante de tempo $k$. A Fig. 4 ilustra a estratégia completa. Então, a função custo deste controlador MBPC é:

$$
\begin{aligned}
J_{2}(k)= & \sum_{j=1}^{N_{\mathrm{y}}}\left(\hat{y}_{\mathrm{T}}(k+j \mid k)-T(k)\right)^{2}+ \\
& +\sum_{j=0}^{N_{\mathrm{u}}-1} \lambda \Delta u(k+j \mid k)^{2}
\end{aligned}
$$

A lei de controle é obtida minimizando a função custo (Eq. 7) em relação a futuras variações no sinal de controle $\Delta u(\cdot)$, sujeito a restrições terminais e do atuador, conforme a Eq. $4 \mathrm{e}$ a estratégia do horizonte rolante, sendo $J(k)=J_{2}(k), \hat{y}(k+$ $j \mid k)=\hat{y}_{\mathrm{T}}(k+j \mid k)$ e $w=T(k)$.

Tal otimização, sem limitações do atuador, tem solução analítica e garantia de estabilidade. No caso com restrições, uma solução numérica pode ser obtida (ver Camacho and Bordons (1999)).

\section{RESULTADOS DE SIMULAÇÃo}

Nesta seção, o desempenho do algoritmo de controle de conforto baseado no índice PMV descrito na Seção 3.1 é analisado. Desta forma, as propriedades da edificação, do sistema de climatização e o modelo são descritas na próxima seção e, na seqüência, o desempenho do sistema em malha-fechada é apresentado.

\subsection{Ambiente de simulação}

Uma edificação (uma zona térmica) cujas dimensões são apresentadas na Fig. 5, foi simulada usando um software de simulação higrotérmica e energética de edificações, PowerDomus (Mendes et al., 2005), baseado em uma formulação a parâmetros concentrados onde, no balanço de energia, são consideradas: cargas de transferência de calor sensível 


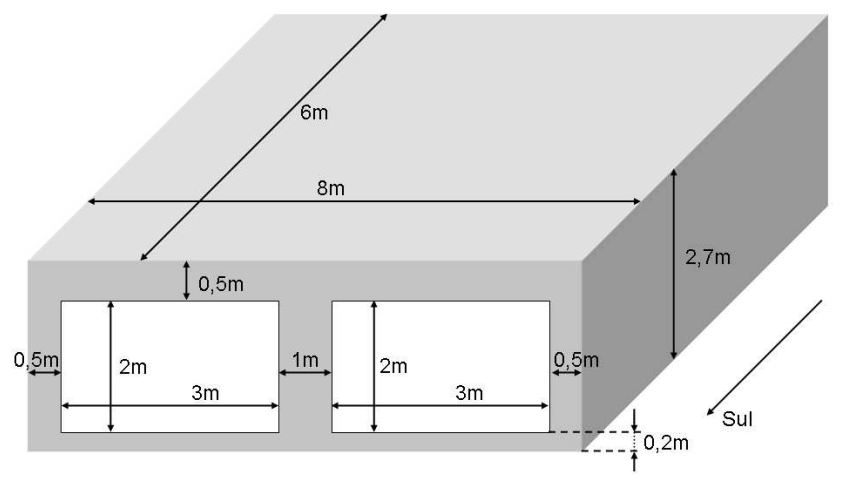

Figura 5: Ambiente de simulação.

e latente por condução, convecção, radiação, infiltração, ventilação, cargas relacionadas ao sistema de climatização dentre outras variáveis. Portanto, o modelo implementado atua como uma edificação real nos experimentos de identificação, sendo os dados climáticos horários (temperatura externa $T_{\text {EXT }}$, umidade relativa externa $\phi_{\text {EXT }}$ e radiação solar global $S_{\mathrm{EXT}}$ ) obtidos de um arquivo de dados climáticos reais da cidade de Curitiba, Brasil.

Assume-se que, dentro da zona térmica, um equipamento de climatização dado por um aquecedor $(5 k W)$ está presente. Assume-se que o aquecedor aceita um sinal de potência continuamente variável entre um mínimo $(0 W)$ e um máximo $(5000 W)$.

Portanto, por meio de um procedimento de identificação de sistemas, as seguintes funções de transferência são obtidas:

$$
\begin{gathered}
\left(1-0,97 q^{-1}\right) y_{\mathrm{T}}(k)=0,08395 q^{-1} u(k) \\
+0,02527 q^{-1} T_{\mathrm{EXT}}(k)+0,2034 q^{-1} \phi_{\mathrm{EXT}}(k) \\
-0,07245 q^{-1} S_{\mathrm{EXT}}(k)+\left(1+0,6767 q^{-1}\right) \xi(k) \\
\left(1-0,9692 q^{-1}\right) y_{\phi}(k)=-0,002107 q^{-1} u(k) \\
+0,0002751 q^{-1} T_{\mathrm{EXT}}(k)+0,01759 q^{-1} H_{\mathrm{EXT}}(k) \\
+0,003342 q^{-1} S_{\mathrm{EXT}}(k)+\left(1+0,7239 q^{-1}\right) \xi(k)
\end{gathered}
$$

sendo $q^{-1}$ o operador atraso unitário. As unidades são: $\left[{ }^{\mathrm{o}} C\right]$ para temperatura e $\left[K W / m^{2}\right]$ para radiação solar. O tempo de amostragem é de 60 segundos. As condições internas da edificação, para calculo do valor do PMV (ver Eq. 2 e Fig. 1), são: velocidade do ar igual a $V=0,1 \mathrm{~m} / \mathrm{s}$ e fatores individuais de atividade igual a $M=1,2$ met e de vestimenta igual a $I_{\mathrm{cl}}=0,66$ clo. Estes valores equivalem a fatores individuais e de atividade comuns de se encontrar em ambientes de escritório. Na seqüência, resultados de malha-fechada são apresentados.

\subsection{Resultados do controle de PMV em malha-fechada}

Dois passos são importantes para a configuração de um controlador preditivo. A definição de um modelo e a seleção dos parâmetros da função custo.

O modelo de previsão para temperatura e umidade relativa é dado por:

$$
\left\{\begin{aligned}
\boldsymbol{x}(k+1)= & {\left[\begin{array}{cc}
0,97 & 0 \\
0 & 0,97
\end{array}\right] \boldsymbol{x}(k)+} \\
& +\left[\begin{array}{c}
0,2431 \\
0,2431
\end{array}\right] u(k) \\
{\left[\begin{array}{c}
\hat{y}_{T}(k) \\
\hat{y}_{\phi}(k)
\end{array}\right]=} & {\left[\begin{array}{cc}
0,3454 & 0 \\
0 & -0,0086
\end{array}\right] \boldsymbol{x}(k) }
\end{aligned}\right.
$$

sendo $\boldsymbol{x}(k)$ os estados do sistema. Neste modelo, o vetor de estados pode ser obtido através dos sinais de saída medidos sem que haja necessidade de estimação de estados. Os parâmetros do modelo foram calculados através de um procedimento de identificação de sistemas baseado em mínimos quadrados, usando modelos com estrutura formada por bases de Laguerre (com pólo em 0,97). Mais detalhes sobre este tipo de modelo para sistemas dinâmicos e sua aplicação em controle preditivo pode ser encontrada em Campello et al. (2007) e Oliveira et al. (2007).

Tal modelo, juntamente com a Eq. 2, são usados para previsão nas estratégias de controle, isto é, com :

$$
\begin{aligned}
\hat{y}_{\mathrm{PMV}}(k)= & G\left(\hat{y}_{\mathrm{T}}(k), \hat{y}_{\phi}(k),\right. \\
& \left.\hat{y}_{\mathrm{T}}(k), V, M, I_{\mathrm{cl}}\right) .
\end{aligned}
$$

onde $V=0,1, M=1,2$ e $I_{\mathrm{cl}}=0,66$. Os parâmetros da função custo são: $N_{\mathrm{y}}=3, N_{\mathrm{u}}=1$ e $\lambda=0$. A janela de restrição é $m=1$.

As simulações são executadas durante um intervalo de tempo de 7 dias, onde o controlador é ligado na hora zero do terceiro dia, isto é, após 48 horas. Dados climáticos externos, que atuam como perturbações na lei de controle, foram coletados de um arquivo de dados climáticos reais da cidade de Curitiba, Brasil da primeira semana de Julho (Inverno) e são apresentados na Fig. 6, onde nota-se claramente a inferência direta da radiação solar na temperatura externa e a relação inversa entre a temperatura e a umidade.

A Fig. 7 apresenta o desempenho da malha-fechada do MBPC com modelo interno de PMV. Nela apresenta-se o sinal de PMV durante o período de simulação. Pode-se notar que este sinal é bastante próximo ao valor ideal de conforto térmico, que é PMV=0. Através da Fig. 7 pode-se ver 

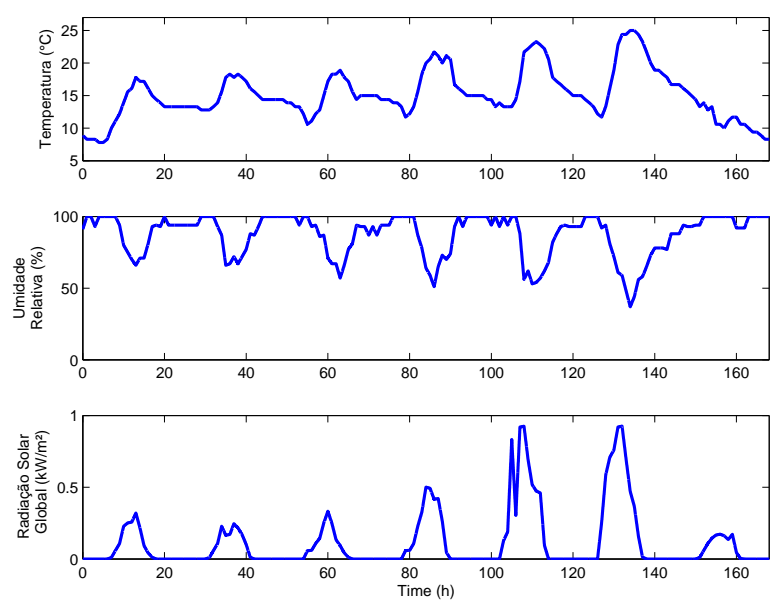

Figura 6: Temperatura externa, umidade relativa e radiação solar global para o período de simulação.

também o comportamento da temperatura interna e do sinal de controle. O consumo de energia é de 206,26 $\mathrm{kWh}$.

\section{RESULTADOS EXPERIMENTAIS}

Nesta seção, o desempenho do algoritmo de controle baseado no índice PMV com geração ótima do sinal de referência é analisado através de um experimento em tempo real. O método é implementado no controle de conforto térmico em uma célula-teste instalada dentro de uma câmara climatizada. Neste tipo de experimento, a câmara térmica é utilizada para representar as condições climáticas externas ao ambiente sob ação do controlador de conforto. Com isso, é possível fazer e reproduzir experimentos com condições externas controladas, o que seria muito difícil em se tratando de condições externas reais. Partes da célula-teste e da câmara climatizada podem ser vistas na Fig. 8. Nesta figura, pode-se perceber a célula no interior da câmara.

A célula possui formato de um cubo de $2 m$ de aresta, suspensa a $0,5 m$ do piso, Resultando em $8 \mathrm{~m}^{3}$ de volume interno e $24 m^{2}$ de área superficial. Para controle de conforto térmico dentro do ambiente, estão presentes dois aquecedores a óleo (potência total de $3000 \mathrm{~W}$ ), três sensores de temperatura e umidade relativa e um sensor de velocidade do ar. A Fig. 9 apresenta a estrutura da célula e a Fig. 10 ilustra o interior da célula com seus aquecedores e sensores.

A implementação do sistema de controle em tempo real é mostrada na Fig. 11. O projeto do controlador é feito $a$ priori utilizando uma ferramenta de projeto, ilustrada aqui pelo software Matlab. Utilizaram-se sensores de temperatura e umidade comerciais, modelo RHT-DM (Novus, 2009a).
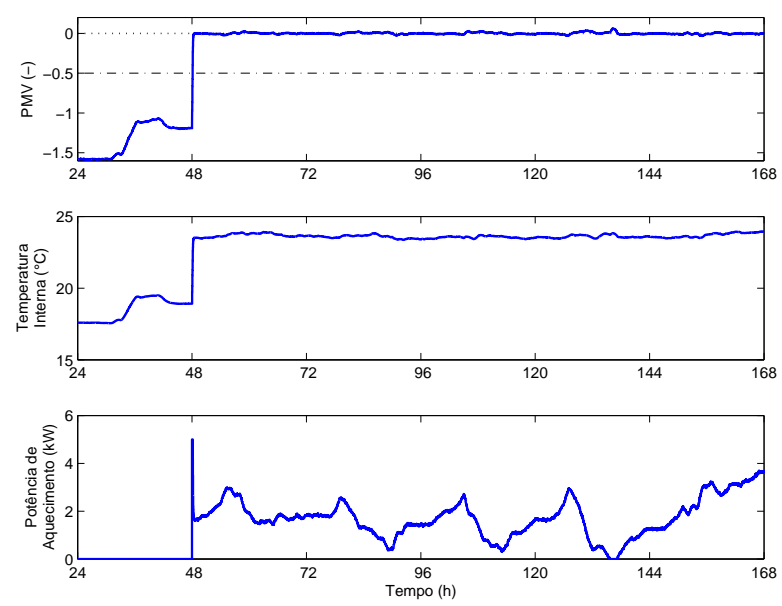

Figura 7: Desempenho do sistema em malha fechada.

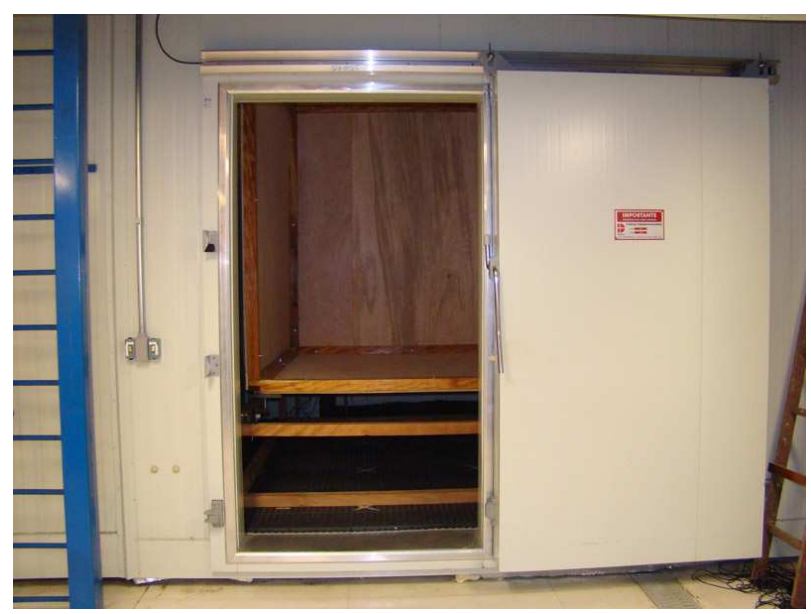

Figura 8: Visão da célula-teste no interior da câmara térmica.

Dentre os sensores de temperatura e umidade, um é utilizado na malha de controle e outro para adquirir informações higrotérmicas em uma posição diferente da utilizada na malha de controle e um terceiro para adquirir dados higrotérmicos externos à célula (dados de temperatura e umidade dentro da câmara climática na qual a célula está inserida). Um sensor comercial, modelo EE66 (Elektronik, 2009) é usado para fazer a medição da velocidade do ar interna. Uma placa comercial de aquisição de dados, modelo NI PCI-6251 (National Instruments, 2009), é utilizada como interface entre os sensores e o computador. A comunicação entre o hardware de controle e o aquecedor é feita através da porta de comunicação RS-232, um circuito tipo PWM (Pulse Width Modulation) e um relé de estado sólido (SSR - Solid State Relay) que detecta passagem pelo nível de tensão $0 \mathrm{~V}$ (modelo N225AC8 (Novus, 2009b)). A freqüência de trabalho escolhida para o 


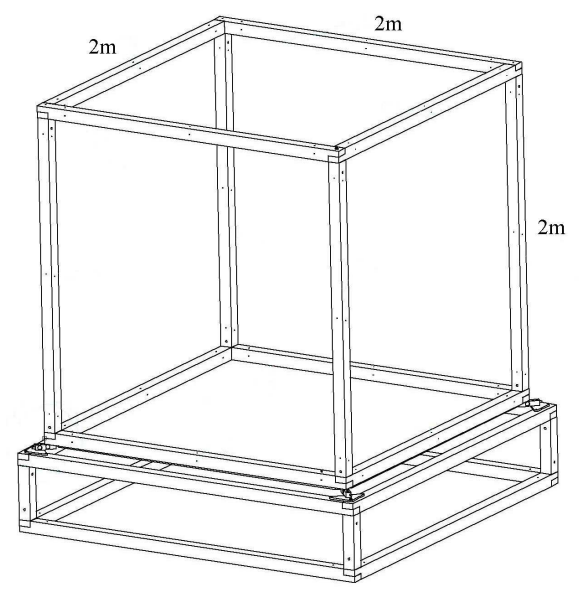

Figura 9: Célula-teste.

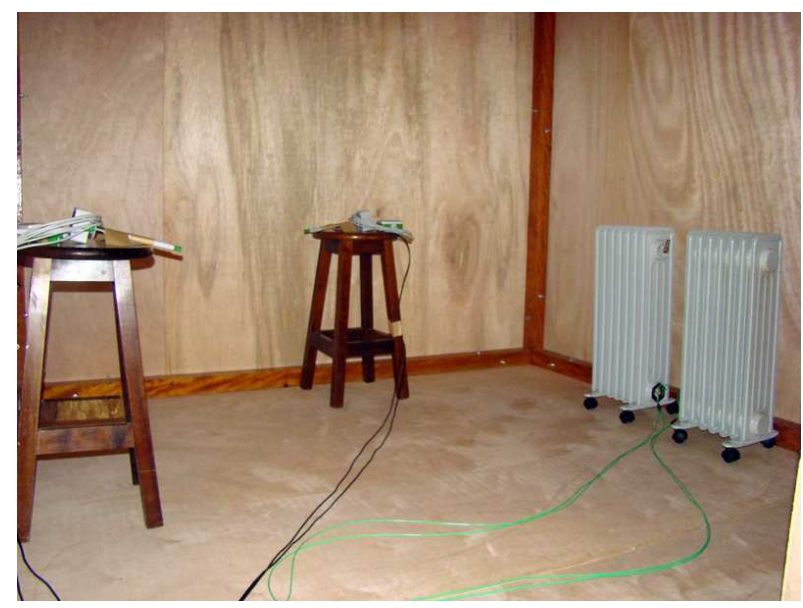

Figura 10: Visão interna da célula-teste.

PWM é de $1 H z$ e, como a tensão de entrada da rede é $60 H z$, tem-se um acionamento do aquecedor em 120 níveis discretos que variam linearmente entre $0 W$ e $3000 W$.

Um software desenvolvido em LabView determina em tempo real o valor ótimo de referência baseado nos parâmetros individuais (taxa metabólica e índice de vestimentas) fornecidos pelo usuário e nos valores medidos de temperatura do ar, umidade relativa e velocidade do ar. Este sinal de referência é utilizado na malha de controle de temperatura, através do algoritmo PMV-MBPC (com modelo externo de PMV), com período de amostragem de 20 segundos.

A especificação do PMV-MBPC é apresentada a seguir. Através de testes de resposta ao degrau, obteve-se uma função transferência de primeira ordem com atraso de transporte para a célula com os aquecedores, como se segue:

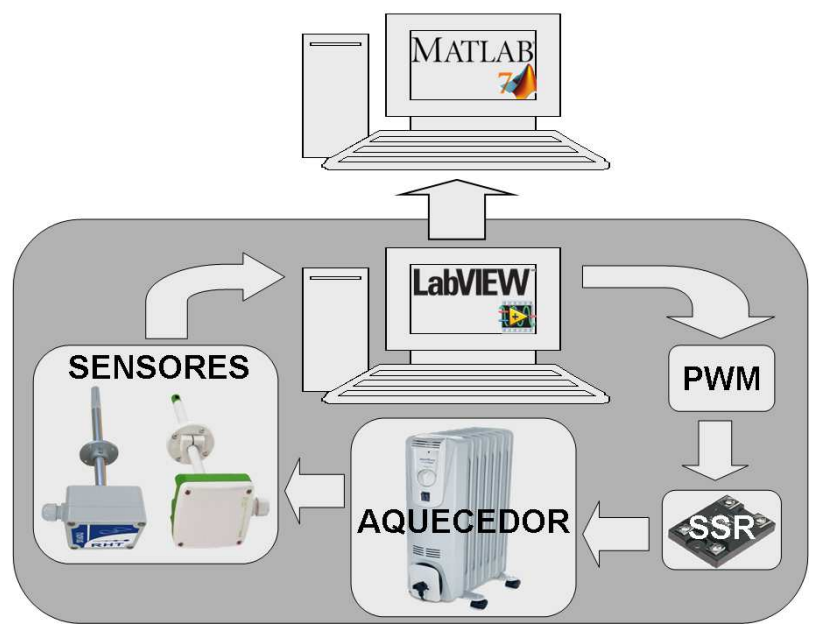

Figura 11: Elementos da malha de controle.
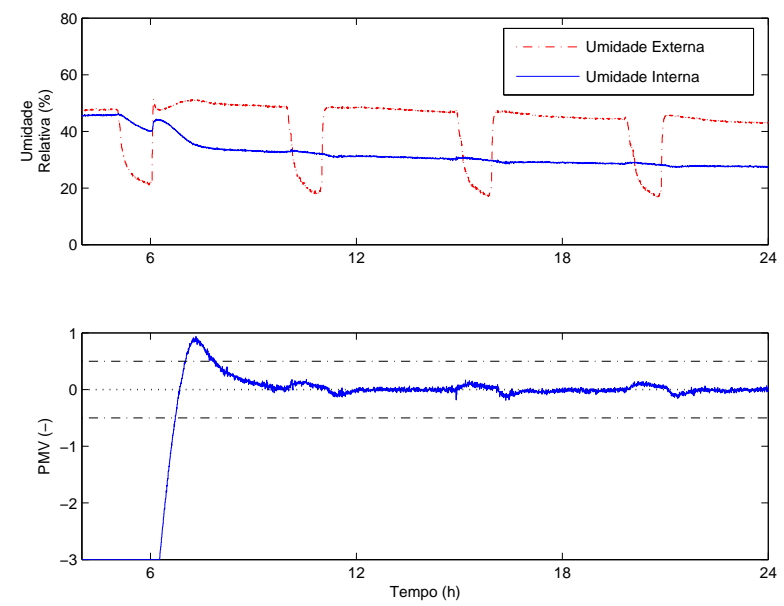

Figura 12: Sinais de umidade relativa e o PMV do ambiente interno.

$$
\left\{\begin{array}{cl}
\boldsymbol{x}(k+1) & =0,9920 \boldsymbol{x}(k)+u(k-28) \\
y_{\mathrm{T}}(k) & =0,1777 \boldsymbol{x}(k)
\end{array}\right.
$$

Os parâmetros de projeto são $N_{\mathrm{y}}=40, N_{\mathrm{u}}=1, \lambda=0$ e $m=1$. Os seguintes parâmetros são assumidos na Eq. 2: $M=1,2$ met e $I_{\mathrm{cl}}=0,75$ clo. A velocidade do ar se manteve em aproximadamente $0,05 \mathrm{~m} / \mathrm{s}$ durante todo o experimento. A umidade relativa interna, necessária para a geração da referência de temperatura, juntamente com a umidade externa à célula, estão apresentadas na Fig. 12.

Neste estudo de caso, a temperatura da câmara onde a célula está inserida é mantida em torno de $10^{\circ} \mathrm{C}$, simulando um dia frio, e a umidade externa é mantida em torno de $50 \%$. O per- 
fil da temperatura externa à célula teste é ilustrado na Fig. 13. Através desta figura e da Fig. 12, oscilações nas temperatura e umidade externas à célula podem ser observadas. Isto é devido ao degelo do evaporador do sistema de climatização da câmara, o que dificulta a ação do sistema de controlador de temperatura e umidade da câmara responsável por manter a temperatura e umidade externa à câmara nos valores desejados.

O desempenho do sistema em malha fechada é ilustrado através da Fig. 12. Pode-se notar que a condição de conforto térmico dentro da célula-teste é alcançada, uma vez que os valores de PMV estão bem próximos ao valor ideal, isto é, o nível 0. De fato, segundo ASHRAE (2001), assumese que valores de PMV entre $-0,5$ e $+0,5$ correspondem a uma situação de conforto. Na Fig. 13, tem-se o seguimento de trajetória da temperatura especificada pelo algoritmo de otimização e o sinal de controle. Portanto, a meta de gerar conforto térmico dentro de um ambiente fechado é obtida, sem a atuação direta de um usuário sobre o sinal de temperatura, mas sim em função de seu padrão de atividade e de vestimenta.

Um segundo experimento é descrito a seguir. Neste experimento, assume-se que a taxa metabólica e o índice de vestimenta do indivíduo dentro da zona térmica variam no tempo conforme apresentado na Fig. 14. Isto significa, inicialmente, o indivíduo está em uma condição de atividade sedentária, vestuário de trabalho, passa para uma atividade equivalente à de estar sentado descansando, vestuário leve, retornando a situação anterior após 6 horas. As condições externas à célula-teste (temperatura e umidade) podem ser visualizadas nas Figs. 15 e 14. Esta é uma das propriedades interessantes do ambiente experimental apresentado, pois condições climáticas semelhantes às do teste anterior puderam ser reproduzidas, o que dificilmente seria possível em ambientes em contato direto com o clima de fato externo.

O controlador aplicado neste teste é o mesmo do teste anterior, assim como as condições de velocidade do ar.

O desempenho do sistema em malha fechada pode ser observado também na Fig. 14, juntamente com a umidade relativa do ar interno e externo. Pode-se notar que a condição de conforto térmico dentro da célula também é alcançada, com um rápido transitório nos momentos de mudança nas condições de atividade e vestimenta. Na Fig. 15, tem-se o seguimento de trajetória da temperatura especificada pelo algoritmo de otimização e o sinal de controle. Nota-se que o sinal de referência de temperatura varia de modo a acompanhar as variações nos parâmetros individuais. Novamente, nota-se que a meta de gerar conforto térmico dentro de um ambiente fechado é obtida, sem a atuação direta sobre o sinal de temperatura, mesmo na presença de variações nos padrões
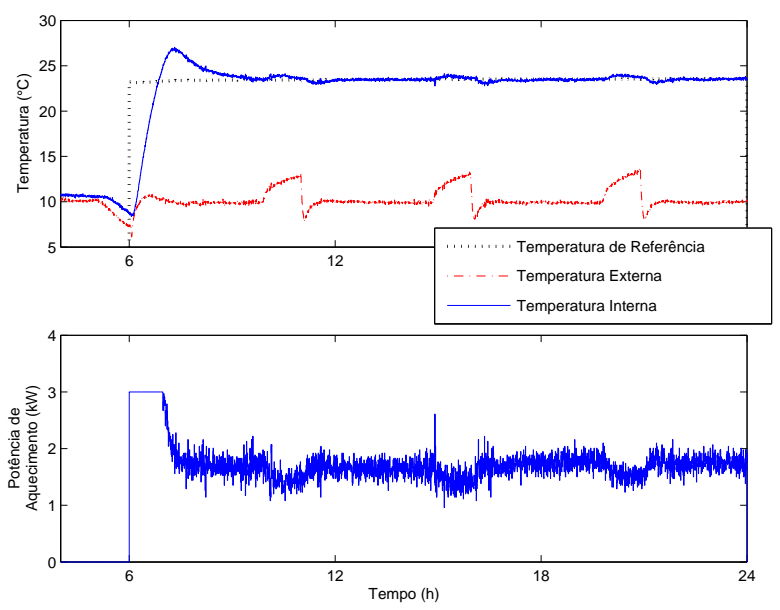

Figura 13: Desempenho do sistema de controle.
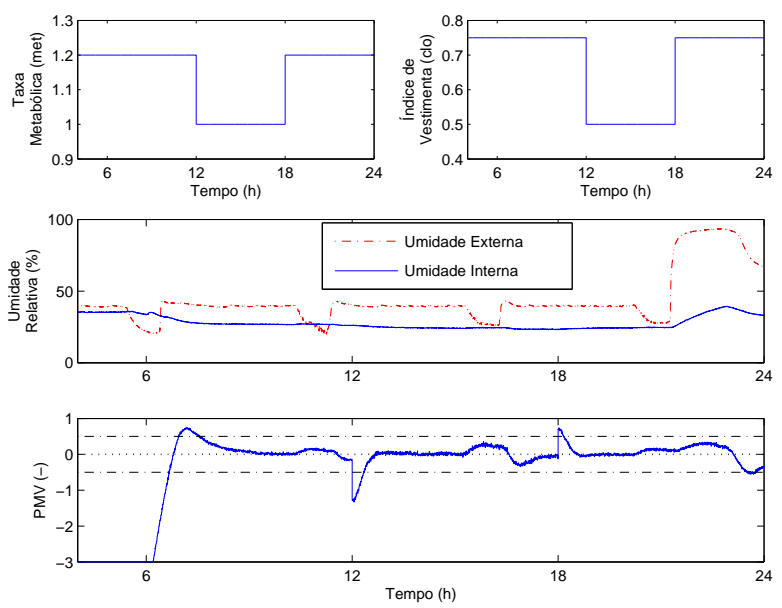

Figura 14: Parâmetros ambientais, individuais e o PMV do ambiente interno.

de atividade e de vestimenta de um indivíduo.

\section{CONCLUSÕES}

Neste artigo, o problema de controle de conforto térmico em edificações (uma única zona térmica) equipadas com um sistema de climatização foi analisado. Dois métodos baseados no MBPC com restrições terminais, caracterizados pelo uso do índice PMV, para otimização das condições de conforto térmico interno foram apresentados.

No primeiro caso, o MPBC com modelo interno de PMV, o índice PMV foi usado para compor o modelo não linear de 


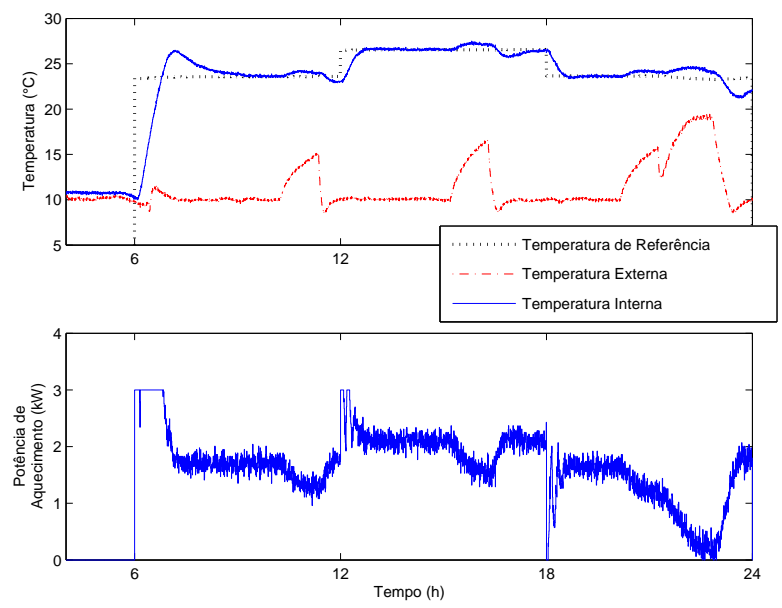

Figura 15: Desempenho do sistema de controle.

previsão com estrutura Wiener em função do sinal de atuação no sistema de climatização. Portanto, tem-se uma estratégia de controle não-linear para conforto térmico, cuja prova de garantia de estabilidade foi apresentada.

No segundo caso proposto, o MPBC com modelo externo de PMV, o índice PMV é usado para gerar um valor ótimo para o sinal de referência. O modelo é linear e uma lei de controle linear foi definida, onde também a estabilidade em malha-fechada é garantida.

Através de resultados numéricos pode-se observar que o algoritmo MPBC com modelo interno de PMV é capaz de controlar conforto térmico em uma edificação. Testes foram realizados usando um modelo baseado em um software de simulação de edificações.

Resultados experimentais em uma célula-teste localizada no interior de uma câmara climática foram também apresentados. Este ambiente se caracteriza por permitir uma atuação nas condições climáticas no exterior do ambiente sob análise e controle, facilitando reproduções e comparações dos resultados obtidos. Pode-se observar que o algoritmo MBPC com modelo externo de PMV foi também capaz de gerar condições de conforto térmico para ocupantes da zona térmica, mesmo na presença de variações nas condições de atividade e vestimenta dos ocupantes. Os níveis de PMV obtidos estiveram dentro do limite aceitável $( \pm 0,5)$, mesmo na presença de perturbações externas.

\section{AGRADECIMENTOS}

Os autores agradecem o apoio da FINEP, através do convênio 01.05.1064.00, e ao CNPq.

\section{REFERÊNCIAS}

Analysis CST (2009). Website. Acesso em 7 de maio de 2009, http://www.labeee.ufsc.br/software/ analysisCst.html.

ASHRAE (2001). Handbook of Fundamentals, American Society of Heating, Refrigerating and Air Conditioning Engineers, Atlanta, GA.

Camacho, E. F. and Bordons, C. (1999). Model Predictive Control in Process Industry, 2 edn, Springer Verlag.

Campello, R. J. G. B., Oliveira, G. H. C. and Amaral, W. C. (2007). Identificação e controle de processos via desenvolvimentos em séries ortonormais. Parte A: identificação, Sba: Controle \& Automação Sociedade Brasileira de Automatica 18(3): 301 - 321.

Castro, M. N., Jota, F. G. and Assis, E. S. (2005). A automação como ferramenta para eficiência energética em edificações, Congresso Brasileiro de Eficiência Energética, Belo Horizonte, Brasil, pp. 268-273.

Clothing, Activity and Human Metabolism (2009). Website. Acesso em 7 de maio de 2009, http://www.engineeringtoolbox.com/ metabolism-clothing-activity-d_117.html.

Clothing and Thermal Insulation (2009). Website. Acesso em 7 de maio de 2009, http://www.engineeringtoolbox.com/ clo-clothing-thermal-insulation-d_732.html.

Donaisky, E. (2008). Avaliação numérica e experimental de técnicas de controle para conforto térmico em edificações, Master's thesis, Pontifícia Universidade Católica do Paraná.

Donaisky, E., Oliveira, G. H. C., Freire, R. Z. and Mendes, N. (2007). PMV-based predictive algorithms for controlling thermal comfort in building plants, Proc. of 16th IEEE Conference on Control Applications (CCA'07), Suntec City, Singapore.

Donaisky, E., Oliveira, G. H. C. and Mendes, N. (2008). Algoritmos PMV-MBPC para conforto térmico em edificações: Aplicação em uma célula-teste, XVII Congresso Brasileiro de Automática (CBA'08), Juiz de Fora, Brazil.

Elektronik (2009). Website. Acesso em 7 de maio de 2009, http://www.epluse.com/uploads/ tx_EplusEprDownloads/manual_EE66_01.pdf.

Erbe, H.-H. (2006). The relevance of energy saving control, Proc. of the IFAC Workshop on Energy Saving Control in Plants and Buildings, Bansko, Bulgaria. 
Fanger, P. O. (1970). Thermal Comfort, McGraw-Hill Inc., New York, USA.

Freire, R. Z., Oliveira, G. H. C. and Mendes, N. (2005a). Análise de algoritmos de controle para sistemas de aquecimento com otimização do conforto térmico, VII Simpósio Brasileiro de Automação Inteligente (SBAI), São Luís, Brasil.

Freire, R. Z., Oliveira, G. H. C. and Mendes, N. (2005b). Thermal comfort based predictive controllers for building heating systems, Proc. of the 16th IFAC World Congress (IFAC'05), Prague, Czech Republic.

Freire, R. Z., Oliveira, G. H. C. and Mendes, N. (2008). Predictive controllers for thermal comfort optimization and energy savings, Energy and Buildings 40: 1353-1365.

FURNAS (2006). Anuário estatístico da companhia furnas centrais elétricas s.a. - ano base 2005, Rua Real Grandeza, 219 - Bloco C - sala 1.603.1 - Rio de Janeiro, Brasil.

Gagge, A. P., Fobelets, A. P. and Berlund, L. G. (1986). A standard predictive index of human response to the thermal environment, ASHRAE Transactions 92: 709-731.

Georgiev, Z. (2006). Benchmarking of HVAC control systems, Proc. of the IFAC Workshop on Energy Saving Control in Plants and Buildings, Bansko, Bulgaria.

Gouda, M. M., Danaher, S. and Underwood, C. P. (2001). Thermal comfort based fuzzy logic controller, Building Serv. Eng. Res. Technol. 22(4): 237-253.

ISO 9237 (1995). Textiles - Determination of the Permeability of Fabrics to Air.

Kolokotsa, D., Tsiavos, D., Stavrakakis, G. S., Lalaitzakis, K. and Antonidakis, E. (2001). Advanced fuzzy logic controllers design and evaluation for buildings occupants thermal-visual comfort and indor air quality satisfaction, Energy and buildings 33: 531-543.

Kon, A. (1994). Thermal comfort sensor, Proc. of the 10th Instrumentation and Measurement Technology Conference, pp. 454-456.

Luna Filho, F. M. and Bauchspiess, A. (2003). Controle fuzzy para automação predial, Controle $e$ Instrumentação 7: 48-54.

Meinander, H. (1985). Introduction of a new test method for measuring heat and moisture transmission trough clothing materials and its application on winter work wear, VTT Publication 24.
Meinander, H. (1994). Thermal properties of clothing systems studied with a sweating thermal manikin, Proc. of 3rd International Symposium on Clothing Comfort Studies, pp. 51-70.

Mendes, N., Oliveira, R. C. L. F. and Santos, G. H. (2005). Energy efficiency and thermal comfort analysis using the powerdomus hygrothermal simulation tool, 9th International IBPSA Conference, Vol. 1, Montréal, Canada, pp. 9-16.

Metabolic Rate Calculator (2009). Website. Acesso em 7 de maio de 2009, http://www.bodyforlife2.com/ calorie_intake.htm.

Muzi, G., Abbritti, G., Accattoli, M. P. and dell'Omo, M. (1998). Prevalence of irritative symptoms in a nonproblem air-conditioned office building, Arch. Occup. Environ. Health (71): 372-378.

National Instruments (2009). Website. Acesso em 7 de maio de 2009, http://sine.ni.com/nips/cds/view/ p/lang/en/nid/14124.

Novus (2009a). Website. Acesso em 7 de maio de 2009, http://www.novus.com.br/catalogos/?ID=645063.

Novus (2009b). Website. Acesso em 7 de maio de 2009, http://www.novus.com.br/catalogos/?ID=519490.

Oliveira, G. H. C., Campello, R. J. G. B. and Amaral, W. C. (2007). Identificação e controle de processos via desenvolvimentos em séries ortonormais. Parte B: controle preditivo, Sba: Controle \& Automação Sociedade Brasileira de Automatica 18(3): 322 - 336.

Prek, M. (2005). Thermodynamic analysis of human heat and mass transfer and their impact on thermal comfort, International Journal of Heat and Mass Transfer 48: 731-739.

Prek, M. (2006). Thermodynamical analysis of human thermal comfort, Energy 31: 732-743.

Raffenel, Y., Blanco, E., Virgone, J., Neveux, P., Scorletti, G. and Thomas, G. (2008). Reducing the energy consumption of space heating in buildings: design of an optimal controller, Proc. of the 17th IFAC World Congress, Seoul, Korea, pp. 11098-11105.

Salsbury, T. I. (2006). Control performance assessment for building automation systems, Proc. of the IFAC Workshop on Energy Saving Control in Plants and Buildings, Bansko, Bulgaria.

Scokaert, P. O. M. and Clarke, D. W. (1994). Stabilising properties of constrained predictive control, IEE Proc. - Control Theory Appl. 141(5): 295-304. 
Sherman, M. (1985). A simplified model of thermal comfort, Energy and Buildings 8: 37-50.

Trebien, R., Oliveira, G. H. C. and Mendes, N. (2007). Sensibilidade do índice PMV e regiões de conforto visando o aperfeiçoamento de climatizadores, Ambiente Construído 7(2): 71-87.

Xavier, A. A. and Lamberts, R. (2000). Indices of thermal comfort developed from field survey in brazil, ASHRAE Transactions 106: 45-58. 